\title{
Factors associated with unsuccessful pessary fitting and reasons for discontinuation in Korean women with pelvic organ prolapse
}

\author{
Sumin Oh, MD, MS $S^{1,{ }^{*}}$, Hye Ryun Namkung, $\mathrm{MD}^{1,{ }^{*}}$, Hee Yeon Yoon, $\mathrm{MD}^{1}$, So Yeon Lee, MD, MS ${ }^{1}$, \\ Myung Jae Jeon, MD, $\mathrm{PhD}^{1,2}$ \\ ${ }^{1}$ Department of Obstetrics and Gynecology, Seoul National University Hospital, Seoul; ${ }^{2}$ Department of Obstetrics and Gynecology, Seoul National \\ University College of Medicine, Seoul, Korea
}

\section{Objective}

To identify the factors associated with unsuccessful pessary fitting and reasons for pessary discontinuation in Korean women with pelvic organ prolapse (POP).

\section{Methods}

The medical records of 234 patients who underwent pessary fitting for the management of symptomatic POP were retrospectively reviewed. A ring pessary with or without support was used. Successful pessary fitting was defined as the ability to wear a pessary for 2 weeks without any discomfort. Factors associated with unsuccessful pessary fitting were determined using a multivariable logistic regression analysis. A Kaplan-Meier survival curve was obtained to examine the probability of continuing pessary use over the follow-up period. The reasons for the discontinuation of pessary were identified.

\section{Results}

Two-hundred-and-twenty-five women were included in the analysis. The rate of unsuccessful pessary fitting was $40 \%$. Prior hysterectomy (odds ratio [OR], 4.13; 95\% confidence interval [CI], 1.81-9.42) and POP quantification stage III-IV $(\mathrm{OR}, 2.49 ; 95 \% \mathrm{Cl}, 1.28-4.85)$ were independent risk factors for unsuccessful pessary fitting. Among the patients with successful pessary fitting, the median time to discontinuation of pessary use was 4 years. The most common causes of discontinued use were vaginal erosion (45.3\%) and urinary incontinence $(26.5 \%)$.

\section{Conclusion}

Patients with a history of hysterectomy and advanced-stage POP are at risk for an unsuccessful fitting of the ring pessary. Vaginal erosion is the main reason for discontinued use among patients with successful pessary fitting.

Keywords: Pelvic organ prolapse; Pessaries

\footnotetext{
Received: 2021.07.16. Revised: 2021.10.04. Accepted: 2021.10.18.

Corresponding author: Myung Jae Jeon, $\mathrm{MD}, \mathrm{PhD}$

Department of Obstetrics and Gynecology, Seoul National University College of Medicine, 103 Daehak-ro, Jongno-gu, Seoul 03080, Korea E-mail: jeonmj@snu.ac.kr

https://orcid.org/0000-0001-5582-1488

*These authors contributed equally to this work.
} 


\section{Obstetrics \& Gynecology Science}

Sumin Oh, et al. Use of a ring pessary in Korean women

\section{Introduction}

Pelvic organ prolapse (POP) is a common gynecological condition that affects approximately half of women over the age of 50 [1]. The prevalence of POP is expected to increase due to several factors, such as aging and obesity in the population [2]. Treatment of symptomatic POP involves surgical or conservative options. A vaginal pessary is a low-risk and costeffective conservative management strategy for POP $[3,4]$. The rates of symptomatic improvement and patient satisfaction after pessary use have been reported to be high [5]. A survey conducted by the American Urogynecologic Society has reported that $77 \%$ of physicians use pessaries as the first-line treatment for POP [6], and a Korean National Health Insurance Database-based cross-sectional study showed that pessary was used in $10 \%$ of all POP patients and $18.5 \%$ of all POP treatments [7].

However, pessaries do not fit well for all women, and there is a certain group of patients who do not benefit from pessary use. The prediction of pessary fitting is beneficial for counseling patients and making decisions regarding treatment. The risk factors for unsuccessful pessary fitting are inconsistent among different studies and include younger age [8], increasing parity [9], higher body mass index [8,10], prior prolapse surgery and/or hysterectomy [9,11-13], advanced prolapse [12], posterior vaginal wall predominant prolapse [12], shorter vaginal length $[10,12-14]$, larger genital hiatus $[12,13]$, and wider vaginal introitus $[12,14]$. There are various pessaries used in different health care centers, which may partly explain the inconsistency in the results among studies.

Even if a pessary is successfully fitted, its use may cause adverse events such as vaginal bleeding, discharge, odor, pain, and urinary incontinence. Any of these factors may lead to the discontinuation of use. It has been reported that $50-80 \%$ of women who were initially successfully fitted continued to use pessaries until one year later [15]. However, data on long-term pessary use are limited.

A ring with or without support is the only type of pessary available in Korea. This study aimed to identify the factors associated with unsuccessful pessary fitting and the reasons for discontinued pessary use in Korean women with POP.

\section{Materials and methods}

We retrospectively reviewed the medical records of 234 patients who underwent pessary fitting trials for the management of symptomatic POP at the Seoul National University Hospital between October 2008 and December 2020. Nine patients who did not visit the clinic after initial pessary insertion were excluded from the analysis. The Institutional Review Board approved this study (SNUH 2106-008-1223), and the requirement for informed consent was waived because of the retrospective nature of the study.

At baseline, demographic and medical history data were collected during an interview, and a standardized pelvic organ prolapse quantification (POP-Q) examination was performed in a $45^{\circ}$ upright sitting position with an empty bladder [16]. As routine clinical care, we offered two ways to manage symptomatic POP with POP-Q stage II or greater (i.e., pessary or surgery) and recommended that patients try pessary insertion first unless they preferred surgical treatment. A ring pessary without support was inserted first, and the largest pessary that was comfortable for the patient was used. If a size up was too uncomfortable for the patient, we used the same size of a ring pessary with support. To check whether the pessary was comfortably fitted, patients were asked to perform Valsalva maneuvers, ambulate, and void her bladder while they were in the office. All patients returned after 2 weeks to assess the fit of the pessary or sooner if there was a problem. Patients were asked whether they experienced any side effects, such as vaginal bleeding, discharge, discomfort, expulsion of the pessary, urinary incontinence, or difficulty during urination or defecation. The pessary was removed and cleaned, and the vagina was examined for erosion. If the pessary fit well and there were no side effects, the pessary was reinserted. Patients who had discomfort or expulsion were offered another pessary if they wanted to continue their use. They returned after 2 weeks and were evaluated again, as described above.

Successful pessary fitting was defined as the ability to wear the pessary for 2 weeks without any discomfort and with up to two insertion attempts. Once successful fitting was confirmed, the patient was educated on how to self-manage the pessary (e.g., removal, cleaning, and reinsertion) and were scheduled to return to the office in 3 months. For patients who could self-manage the pessary, scheduled in-person follow-up was performed every 6 months thereafter. For 


\section{Obstetrics \& Gynecology Science}

Vol. 65, No. 1, 2022

those who were not able to self-manage the pessary, followup was recommended every 3 months. At each visit, the patients were asked about any untoward symptoms and underwent a speculum examination for vaginal erosion. When moderate to severe erosion was observed, a pessary-free rest period of 4 weeks was taken, and the patient was advised to use topical estrogen. Thereafter, the pessary was replaced if healing had occurred. All medical practices were performed by a urogynecology specialist (M.J.J.).

Data were analyzed using the SPSS software (version 26; SPSS Inc., Chicago, IL, USA). The normality of the data was assessed using the Shapiro-Wilk test. Comparisons between the groups for continuous variables were performed using the two-sample $t$-test or Mann-Whitney $U$ test. To compare the categorical variables between the groups, the chi-squared test or Fisher's exact test was performed. After univariable analysis, the variables with $P$-values of $<0.05$ were included in a multivariable analysis using logistic regression. A Kaplan-
Meier survival plot was obtained to examine the probability of continued pessary use over the follow-up period. A $P$-value of $<0.05$ was considered statistically significant.

\section{Results}

Two-hundred and twenty-five women were included in the final analysis, with a median age of 73 years. The median body mass index of the study population was $24.4 \mathrm{~kg} / \mathrm{m}^{2}$. Most women were postmenopausal and presented with an advanced stage of POP (POP-Q stage III or IV). Thirty-two women $(14.2 \%)$ had a history of hysterectomy.

Table 1 presents the characteristics of the patients with successful and unsuccessful pessary fitting. The rate of unsuccessful pessary fitting was $40 \%$. Multivariable logistic regression analysis showed that prior hysterectomy (odds ratio [OR], 4.13; 95\% confidence interval $[\mathrm{Cl}], 1.81-9.42)$ and POP-Q

Table 1. Univariable and multivariable analyses of the risk factors of unsuccessful ring pessary fitting

\begin{tabular}{|c|c|c|c|c|c|c|}
\hline \multirow{2}{*}{ Characteristic } & \multirow{2}{*}{ Total $(n=225)$} & \multirow{2}{*}{$\begin{array}{c}\text { Successful } \\
(n=135)\end{array}$} & \multirow{2}{*}{$\begin{array}{l}\text { Unsuccessful } \\
\qquad(n=90)\end{array}$} & \multicolumn{2}{|c|}{$P$-value } & \multirow{2}{*}{ OR $(95 \% \mathrm{Cl})$} \\
\hline & & & & Univariable & Multivariable & \\
\hline Age (yr) & 73.0 (30.0 to 95.0) & 73.0 (30.0 to 87.0) & 72.0 (31.0 to 95.0) & 0.197 & & \\
\hline Body mass index $\left(\mathrm{kg} / \mathrm{m}^{2}\right)$ & $24.4 \pm 3.1$ & $24.5 \pm 3.3$ & $24.4 \pm 2.8$ & 0.765 & & \\
\hline Vaginal parity & $3.0(0.0$ to 8.0$)$ & $3.0(0.0$ to 8.0$)$ & $3.0(0.0$ to 5.0$)$ & 0.082 & & \\
\hline Postmenopause & $216(96.0)$ & $131(97.0)$ & $85(94.4)$ & 0.331 & & \\
\hline Prior hysterectomy & $32(14.2)$ & $11(8.1)$ & $21(23.3)$ & 0.001 & $<0.001$ & $4.13(1.81-9.42)$ \\
\hline Prior prolapse surgery & $23(10.2)$ & $12(8.9)$ & $11(12.2)$ & 0.419 & & \\
\hline POP-Q stage & & & & 0.022 & 0.004 & $2.49(1.28-4.85)$ \\
\hline$\|$ & $64(28.4)$ & $46(34.1)$ & $18(20.0)$ & & & \\
\hline III-IV & $161(71.6)$ & $89(65.9)$ & $72(80.0)$ & & & \\
\hline Leading compartment & & & & 0.139 & & \\
\hline Anterior & $157(69.8)$ & $100(74.1)$ & $57(63.3)$ & & & \\
\hline Apical & $59(26.2)$ & $29(21.5)$ & $30(33.3)$ & & & \\
\hline Posterior & $9(4.0)$ & $6(4.4)$ & $3(3.3)$ & & & \\
\hline \multicolumn{7}{|l|}{ POP-Q values } \\
\hline $\mathrm{Ba}$ & $2.0(-2.5$ to 8.0$)$ & $2.0(-2.5$ to 7.5$)$ & $2.0(-1.0$ to 8.0$)$ & 0.891 & & \\
\hline C & $1.0(-8.0$ to 8.0$)$ & $0.5(-6.0$ to 7.5$)$ & $1.0(-8.0$ to 8.0$)$ & 0.362 & & \\
\hline $\mathrm{Bp}$ & $0.0(-3.0$ to 8.0$)$ & $0.0(-3.0$ to 7.5$)$ & $0.0(-3.0$ to 8.0$)$ & 0.460 & & \\
\hline Genital hiatus & $5.0(2.0$ to 8.0$)$ & $5.0(2.0$ to 8.0$)$ & 5.0 (3.0 to 8.0$)$ & 0.526 & & \\
\hline Perineal body & $3.0(1.0$ to 6.0$)$ & $3.0(1.0$ to 6.0$)$ & $3.0(2.0$ to 5.0$)$ & 0.056 & & \\
\hline Total vaginal length & 7.0 (5.5 to 9.0$)$ & $7.0(6.0$ to 9.0$)$ & $7.0(5.5$ to 9.0$)$ & 0.382 & & \\
\hline
\end{tabular}

Values are presented as mean \pm standard deviation, median (range), or number (\%).

$\mathrm{OR}$, odds ratio; $\mathrm{Cl}$, confidence interval; POP-Q, pelvic organ prolapse quantification. 


\section{Obstetrics \& Gynecology Science}

Sumin Oh, et al. Use of a ring pessary in Korean women

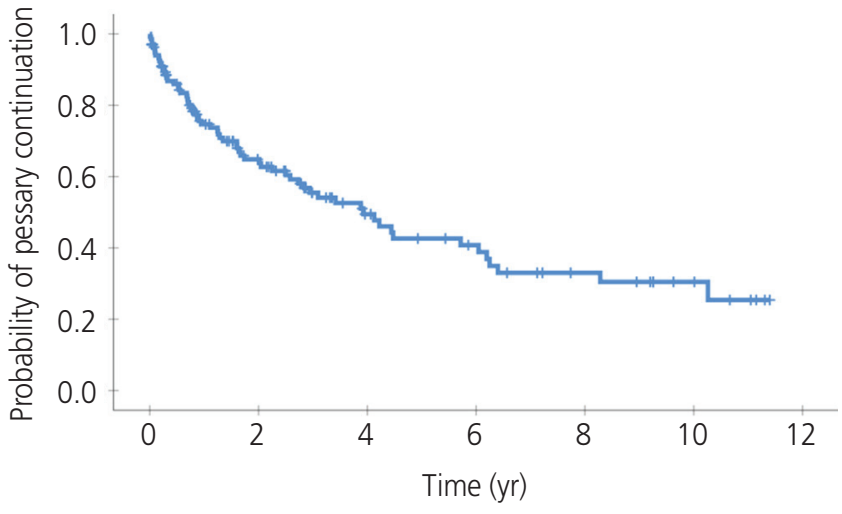

Fig. 1. Kaplan-Meier graph showing the probability of continued pessary use.

stage III-IV (OR, 2.49; 95\% Cl, 1.28-4.85) were independent risk factors for unsuccessful pessary fitting.

The median follow-up duration for the successful pessary fitting group was 27 months (range, 0.5-141). Fig. 1 shows a Kaplan-Meier plot that presents the probability of continued pessary use. One year after successful pessary fitting, the probability of continued pessary use was $74.7 \%$, and the median time to discontinuation of use was 4 years. Fig. 2 shows the reasons for discontinued pessary use, and the most common cause was vaginal erosion (45.3\%), followed by urinary incontinence (26.5\%). Among 64 women who discontinued pessary use during their follow-up period, $60.3 \%$ underwent surgery afterward, and $39.7 \%$ chose observation or were lost to follow-up.

\section{Discussion}

The current study showed that prior hysterectomy and advanced-stage POP were associated with unsuccessful ring pessary fitting. The rate of successful pessary fitting was $60 \%$, and half of these patients continued their use for 4 years. For women who discontinued pessary use, the most common cause was vaginal erosion.

Successful pessary fitting has a heterogeneous definition according to the literature, and most studies reported the rates of successful pessary fitting with the use of several types of pessaries. When successful fitting was defined as the ability to retain the pessary for 1 to 3 weeks without any discomfort, the rates of successful pessary fitting were $41 \%$ to $88 \%$ [8-14], and our results lie within that range. Consistent

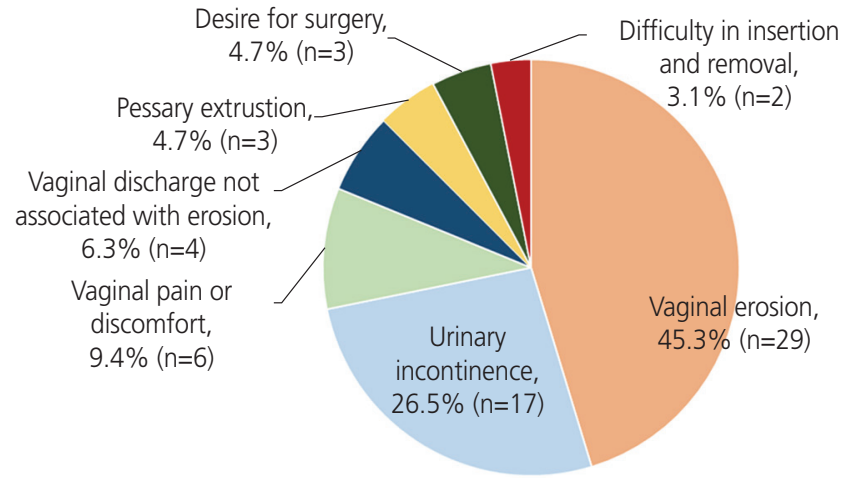

Fig. 2. Reasons for discontinuation of pessary use $(n=64)$.

with our findings, several studies have reported that prior hysterectomy is a risk factor for unsuccessful fitting $[9,11,13]$. A possible explanation is that the protruding cervix may play a role in stabilizing the ring pessary. Furthermore, there may be shortening or narrowing of the vaginal apex after hysterectomy, making it difficult to retain the pessary. On the other hand, most studies have reported that the severity of POP is not associated with unsuccessful pessary fitting [8-11,13,14]. However, these studies used space-occupying pessaries, such as a Gellhorn pessary, in cases where the ring pessary did not stay in place. One study in which a stratified analysis was performed according to the type of pessary, found that a higher POP stage was an independent risk factor for unsuccessful ring pessary fitting [12]. To retain a ring pessary, the internal vaginal caliber must be wider than the vaginal opening [17]. Patients with advanced-stage POP tend to have a wide introitus; therefore, they are more likely to experience pessary expulsion. Space-occupying pessaries, such as the Gellhorn pessary, function by creating more friction and suction within the vagina; therefore, they are less affected by the width of the vaginal introitus than the ring pessary [18].

Only a few studies have evaluated the long-term continuation of pessary use. Continuation rates at 5-7 years range from $14 \%$ to $86 \%$, and the main reasons for discontinuation were complications arising from pessary use rather than ineffectiveness [19-21]. In our cohort, the median time to pessary discontinuation was 4 years, and vaginal erosion was the most common cause of discontinuation. A recent systematic review found that the most common pessary complication was vaginal erosion [22]. Local pressure from the pessary can lead to focal devascularization and vaginal erosion. Reported risk factors for vaginal erosion include long-term uninterrupted use and placement of a pessary that is too large [22]. 


\section{Obstetrics \& Gynecology Science}

Vol. 65 , No. 1, 2022

Proper sizing and periodic examinations may help reduce vaginal erosion and the resultant discontinuation of pessary use.

To the best of our knowledge, this is the first study to report the risk factors associated with unsuccessful pessary fitting and reasons for discontinuation of use in Korean women. Nonetheless, there were some limitations, mainly arising from the inherent limitations of the retrospective study design. For instance, a selection bias may have existed in the study population. Patients who chose to undergo surgery at the initial visit were excluded from the pessary fitting trial. Different outcomes could arise if all patients with POP were made to try a pessary first, regardless of their discretion. However, this type of practice might be unrealistic. Additionally, we had limited pessary choices, so the findings may not be suitable for the use of other types of pessaries such as the Gellhorn or donut pessary.

In conclusion, patients with a history of hysterectomy and advanced POP are at risk of unsuccessful ring pessary fitting. Vaginal erosion was the main reason for discontinuation in patients with successful pessary fitting. In light of the fact that rings with or without support are the only type of pessary available in Korea, the results from our study would be beneficial for counseling patients and making decisions regarding treatment.

\section{Conflict of interest}

No potential conflict of interest relevant to this article was reported.

\section{Ethical approval}

The Institutional Review Board approved this study (SNUH 2106-008-1223).

\section{Patient consent}

The requirement for informed consent was waived because of the retrospective nature of the study.

\section{Funding information}

None.

\section{References}

1. Hagen S, Stark D. Conservative prevention and management of pelvic organ prolapse in women. Cochrane Database Syst Rev 2011;(12):CD003882.

2. Nygaard I, Barber MD, Burgio KL, Kenton K, Meikle $S$, Schaffer J, et al. Prevalence of symptomatic pelvic floor disorders in US women. JAMA 2008;300:1311-6.

3. Lone F, Thakar R, Sultan AH. One-year prospective comparison of vaginal pessaries and surgery for pelvic organ prolapse using the validated ICIQ-VS and ICIQ-UI (SF) questionnaires. Int Urogynecol J 2015;26:1305-12.

4. Sung VW, Wohlrab KJ, Madsen A, Raker C. Patient-reported goal attainment and comprehensive functioning outcomes after surgery compared with pessary for pelvic organ prolapse. Am J Obstet Gynecol 2016;215:659.e17.

5. Bai SW, Yoon BS, Kwon JY, Shin JS, Kim SK, Park KH. Survey of the characteristics and satisfaction degree of the patients using a pessary. Int Urogynecol J Pelvic Floor Dysfunct 2005;16:182-6; discussion 186.

6. Cundiff GW, Weidner AC, Visco AG, Bump RC, Addison WA. A survey of pessary use by members of the American Urogynecologic Society. Obstet Gynecol 2000;95(6 Pt 1):931-5.

7. Yuk JS, Lee JH, Hur JY, Shin JH. The prevalence and treatment pattern of clinically diagnosed pelvic organ prolapse: a Korean National Health Insurance Database-based cross-sectional study 2009-2015. Sci Rep 2018;8:1334.

8. Panman CM, Wiegersma M, Kollen BJ, Burger $\mathrm{H}$, Berger MY, Dekker JH. Predictors of unsuccessful pessary fitting in women with prolapse: a cross-sectional study in general practice. Int Urogynecol J 2017;28:307-13.

9. Fernando RJ, Thakar R, Sultan AH, Shah SM, Jones PW. Effect of vaginal pessaries on symptoms associated with pelvic organ prolapse. Obstet Gynecol 2006;108:93-9.

10. Mao M, Ai F, Zhang Y, Kang J, Liang $S$, Xu T, et al. Predictors for unsuccessful pessary fitting in women with symptomatic pelvic organ prolapse: a prospective study. 


\section{Obstetrics \& Gynecology Science}

Sumin Oh, et al. Use of a ring pessary in Korean women

BJOG 2018;125:1434-40.

11. Mutone MF, Terry C, Hale DS, Benson JT. Factors which influence the short-term success of pessary management of pelvic organ prolapse. Am J Obstet Gynecol 2005;193:89-94.

12. Ma C, Xu T, Kang J, Zhang Y, Ma Y, Liang S, et al. Factors associated with pessary fitting in women with symptomatic pelvic organ prolapse: a large prospective cohort study. Neurourol Urodyn 2020;39:2238-45.

13. Markle D, Skoczylas L, Goldsmith C, Noblett K. Patient characteristics associated with a successful pessary fitting. Female Pelvic Med Reconstr Surg 2011;17:249-52.

14. Clemons JL, Aguilar VC, Tillinghast TA, Jackson ND, Myers DL. Risk factors associated with an unsuccessful pessary fitting trial in women with pelvic organ prolapse. Am J Obstet Gynecol 2004;190:345-50.

15. Lamers BH, Broekman BM, Milani AL. Pessary treatment for pelvic organ prolapse and health-related quality of life: a review. Int Urogynecol J 2011;22:637-44.

16. Bump RC, Mattiasson A, Bø K, Brubaker LP, DeLancey JO, Klarskov $P$, et al. The standardization of terminology of female pelvic organ prolapse and pelvic floor dysfunction. Am J Obstet Gynecol 1996;175:10-7.

17. Culligan PJ. Nonsurgical management of pelvic organ prolapse. Obstet Gynecol 2012;119:852-60.

18. Ding J, Song XC, Deng M, Zhu L. Which factors should be considered in choosing pessary type and size for pelvic organ prolapse patients in a fitting trial? Int Urogynecol J 2016;27:1867-71.

19. Broens-Oostveen MC, Mom RM, Lagro-Janssen AL. Genital prolapse; treatment and course in four general practices. Ned Tijdschr Geneeskd 2004;148:1444-8.

20. Sarma S, Ying T, Moore KH. Long-term vaginal ring pessary use: discontinuation rates and adverse events. BJOG 2009;116:1715-21.

21. Lone F, Thakar R, Sultan AH, Karamalis G. A 5-year prospective study of vaginal pessary use for pelvic organ prolapse. Int J Gynaecol Obstet 2011;114:56-9.

22. Abdulaziz M, Stothers L, Lazare D, Macnab A. An integrative review and severity classification of complications related to pessary use in the treatment of female pelvic organ prolapse. Can Urol Assoc J 2015;9:E400-6. 